\title{
Caracterización morfoagronómica y evaluación del rendimiento de doce genotipos de pallar (Phaseolus lunatus L.) de granos de colores en la zona media del Valle de Ica
}

\author{
Luz Marina Espinoza de Arenas \\ luz.espinoza@unica.edu.pe
}

Pedro Antonio Aquije Gómez

pedro.aquije@unica.edu.pe

Felipe Artemio Surco Laos

felipe.surco@unica.edu.pe

\section{Guillermo Espino Tipismana \\ guillermo.espino@gmail.com}

Yasmin Rojas Yaba

20160126@unica.edu.pe

Universidad Nacional "San Luis Gonzaga"

Ica - Perú.

\section{RESUMEN}

Con la finalidad de evaluar genotipos de Phaseolus lunatus de granos de color, colectados en diversos lugares de la región Ica y el país, se planificó realizar la caracterización morfoagronómica y el comparativo de rendimiento de doce genotipos según el diseño en bloques completamente al azar, en condiciones de suelo y clima de la zona media del valle de Ica, distrito de Subtanjalla, de marzo a octubre del 2021, con las restricciones propias de la pandemia mundial. Las plantas de pallar mostraron buena adaptación, siendo todas de hábito de crecimiento indeterminado. Se ha encontrado diferencia altamente significativa entre tratamientos para longitud de la guía principal, días al $50 \%$ de floración, número de granos por vaina, peso de 100 granos, días a madurez de la vaina, peso de grano por planta, largo y ancho de la vaina, largo y ancho del grano. La variabilidad de las características del grano se expresó en color, tamaño y forma. Se encontró alta correlación positiva entre la longitud de la guía principal y la cobertura de follaje, número de vainas por planta, peso de 100 granos, ancho de vaina y peso de grano por planta y se ha encontrado material segregante que es necesario evaluar.

Palabras clave: phaseolus lunatus; caracterización; pallar nativo; rendimiento 


\title{
Morphoagronomic characterization and evaluation of the yield of twelve genotypes of lima bean (Phaseolus lunatus $\mathbf{L}$.) of colored grains in the middle zone of the Ica Valley
}

\begin{abstract}
In order to evaluate Phaseolus lunatus genotypes of colored grains, collected in various places in the Ica region and the country, it was planned to carry out the morphoagronomic characterization and the yield comparison of twelve genotypes according to the completely randomized block design, in Soil and climate conditions of the middle zone of the Ica valley, Subtanjalla district, from March to October 2021, with the restrictions of the global pandemic. Lima bean plants showed good adaptation, all being of indeterminate growth habit. A highly significant difference has been found between treatments for length of the main guide, days at $50 \%$ flowering, number of grains per pod, weight of 100 grains, days to pod maturity, grain weight per plant, length and width of the pod, length and width of the grain. The variability of the characteristics of the grain was expressed in color, size and shape. A high positive correlation was found between the length of the main guide and the foliage cover, number of pods per plant, weight of 100 grains, pod width and grain weight per plant, and segregating material that needs to be evaluated has been found.
\end{abstract}

Keywords: phaseolus lunatus; characterization; native lima bean; yield

Artículo recibido: 30 noviembre. 2021 Aceptado para publicación: 29 diciembre 2021 Correspondencia: luz.espinoza@unica.edu.pe

Conflictos de Interés: Ninguna que declarar 


\section{INTRODUCCIÓN}

Phaseolus lunatus L. tiene dos centros de domesticación independientes, uno en Mesoamérica y otro más reciente en los Andes en América del Sur, en cada uno de dichos centros se encuentran tanto variedades silvestres como cultivadas y constituyen el pool genético primario de la especie (Fofana et al., 2001).

El género Phaseolus (subfase Phaseolinae, tribu Phaseoleae, familia Leguminosae), tiene una historia taxonómica y nomenclatura compleja, que es bien ilustrado por P. lunatus, el frijol lima - pallar (Maquet, 1995; Baudoin et al., 2004). Las investigaciones apoyan firmemente un Phaseolus monofilético; Phaseolus es estrictamente de origen del Nuevo Mundo, concentrado en América tropical templada y cálida. Probablemente hay Alrededor de 50 especies del género, de las cuales cinco son domesticadas: frijol común (P. vulgaris L.), frijol lima (P. lunatus L.), frijol escarlata (P. coccineus L.), tepary frijol (P. acutifolius A. Gray) y frijol de año (P. polyanthus Greenm.) (Debouck, 1999).

P. lunatus pudo originarse en la región neotropical de América, desde México y América Central, hasta la región andina de Perú, Chile y la zona central de América del Sur. Se cree que su centro de origen se encuentra en Guatemala, por haberse hallado al progenitor silvestre de esta especie en ese país. Por otra parte, existen estudios que proponen, incluso con evidencias moleculares, que su origen principal es la región andina y que su distribución ancestral por toda América se dio por domesticación. Su distribución silvestre y su domesticación son similares a los de Phaseolus vulgaris. Ambas especies fueron domesticadas independientemente en Mesoamérica y en la zona andina, a partir de especies progenitoras silvestres de amplia distribución. P. lunatus fue domesticada en dos centros principales: en la región andina, hace unos 4000 años, en la parte occidental de la cordillera de los Andes, desde el norte de Perú a Ecuador y Bolivia; y en la región mesoamericana, hace unos 3000 años, principalmente desde México a Costa Rica, e incluso a través de la región oriental de los Andes llegando al norte de Argentina. Estas domesticaciones dieron origen a dos ecotipos: sieva o mesoamericano, de granos pequeños; y Lima o andino, de granos grandes. El mesoamericano corresponde a las líneas de dispersión hopi, entre América Central y los Estados Unidos, y Caribe, que abarca las Antillas; mientras que el andino corresponde a la línea de dispersión inca (FAO, 2018).

Los valles de la región Ica, reúnen condiciones agroecológicas apropiadas para cultivar 
el pallar en sus diferentes variedades, las de hábito de crecimiento indeterminado postrado o semi postrado o determinado; siendo una interesante alternativa económica para los productores, pues ahora más que antes, pueden colocar sus granos a mejores precios, ya que esta menestra es fuente importante de proteína vegetal; sin embargo tiene factores limitantes debido a un inadecuado manejo del suelo, del agua, de los nutrientes, de las plagas y enfermedades (Espinoza, 2012).

Los caracteres morfoagronómicos corresponden a los caracteres morfológicos que son relevantes en la utilización de las especies cultivadas. Pueden ser de tipo cualitativo o cuantitativo, e incluyen algunos de los caracteres botánicos-taxonómicos más otros que no necesariamente identifican la especie, pero que son importantes desde el punto de vista de necesidades agronómicas, de mejoramiento genético, y de mercadeo y consumo. A manera de ejemplos de estos caracteres se puede mencionar la forma de las hojas; pigmentaciones en raíz, tallo, hojas y flores; color, forma y brillo en semillas; tamaño, forma y color de frutos; arquitectura de planta expresada en hábito de crecimiento y tipos de ramificación (Franco e Hidalgo, 2003).

Como antecedentes internacionales, se tiene que Guimaraes et al (2007), realizaron el análisis de la diversidad genética de 22 accesiones de frijol-fava ( $P$. lunatus $L$.) oriundos de los Estados de Ceará, Paraíba y Pernambuco - Brasil utilizando marcadores RAPD y catorce de estos genotipos fueron caracterizados a través de marcadores morfológicos. Para caracterizar la variabilidad genética utilizaron 60 loci polimórficos. Al analizar la muestra observaron la formación de dos grupos y cuatro subgrupos, notando una alta variabilidad genética entre las accesiones. Encontraron que los genotipos más cercanos genéticamente fueron FA-01 y FA-02, del estado de Ceará, con 85\% de similitud, y los menos similares fueron FA-07 y FA-20, de los estados de Ceará y Pernambuco, respectivamente, con 35,9\% de similitud. En cuanto a la caracterización morfológica, encontraron que el genotipo FA-13 en comparación con otros presentó valores más altos de peso de semilla, número de semillas por vaina, largo y ancho de vaina, mientras que el genotipo FA-16 mostró valores menores. Para el peso de cien semillas, semillas muy pequeñas, menor número de vainas por planta, menor longitud de vaina y menor producción de semilla por planta.

Para evaluar la variabilidad de poblaciones silvestres de P. lunatus L. originarias del occidente de México, (López-Alcocer et al., 2016) realizaron la caracterización 
morfológica de 30 colectas, en diez plantas seleccionadas al azar con 25 descriptores cualitativos y diez cuantitativos. A las variables cuantitativas les calculó el coeficiente de correlación, análisis de agrupamiento y componentes principales. Encontraron correlación positiva alta entre el peso de 100 semillas y longitud y ancho de hoja primaria, longitud de vaina y altitud de colecta. El análisis de conglomerados conformó dos grupos: el grupo A de floración tardía y el grupo B de floración precoz, y dentro de cada grupo se detectaron los subgrupos A1 y B1 de semillas pequeñas procedentes de localidades de baja altitud y los subgrupos A2 y B2 de semillas grandes originarios de localidades de mayor altitud.

En el Perú, Pesantes et al. (2015), refieren que determinaron la variabilidad morfoagronómica de ocho poblaciones de P. lunatus L. "pallar" procedentes de Trujillo (costa) y Huamachuco (sierra del Perú), cultivadas bajo condiciones agro-ecológicas de la costa norperuana. Señalan que utilizaron la codificación PLUNT 01 a 08 y analizaron seis caracteres cuantitativos que fueron procesados estadísticamente. Encontraron marcadas diferencias fenotípicas y genéticas entre las ocho colectas cultivadas, como en el carácter días a la floración, peso de los granos y número de semillas por vaina.

A nivel local, Espinoza y Espino (2017), informan que realizaron la evaluación morfológica, fenológica y de componentes de rendimiento de 30 genotipos de pallar de hábito de crecimiento indeterminado en el distrito de Pachacútec, zona baja del valle de Ica, en diez plantas marcadas. Encontraron que los componentes morfológicos como color de flor, forma de la vaina, forma y tamaño del grano, siendo variables cualitativas, tuvieron un comportamiento definido sin influencia del ambiente; el color de flor fue blanco o lila, predominando el color lila como la variedad testigo. Señalan que la mayoría de genotipos evaluados fueron más precoces que la variedad testigo; pero en componentes de rendimiento destacó la variedad testigo con 414,83 g/planta (4 718,33 kg ha¹), ubicándose en el primer lugar; otros 16 genotipos lograron buenos rendimientos con promedios por encima de $3000 \mathrm{~kg} \mathrm{ha}^{-1}$.

De igual manera, Espinoza y Espino (2020), señalan que realizaron la caracterización morfológica de 20 genotipos de pallar de hábito de crecimiento indeterminado, en la zona media del valle de Ica en condiciones edafoclimáticas favorables para el cultivo, evaluando once caracteres cuantitativos y siete morfológicos. Informan que hallaron el promedio, desviación estándar y el coeficiente de variación de cada una de las variables 
cuantitativas evaluadas, encontraron alta correlación positiva con $(r>0.56)$ entre las dimensiones del grano con las dimensiones de la vaina; entre el peso de 100 semillas con el largo de vaina, ancho de vaina, largo de grano y ancho de grano; entre los días al $50 \%$ de floración con el número de vainas por planta; entre la madurez de la vaina con el número de vainas por planta y los días al 50 \% de la floración; entre el peso de grano por planta con el número de vainas por planta, largo de vaina, ancho de grano, peso de 100 semillas, días al 50\% de la floración y los días a la madurez de la vaina. Señalan que a un coeficiente de distancia genética de 0.6, los genotipos de pallar conformaron siete grupos bien definidos, mostrando una importante diversidad de pallar expresada en los días a la floración, madurez de la vaina y en los principales componentes de rendimiento. En cuanto a las variables morfológicas, color de flor, curvatura de la vaina, forma del ápice de la vaina, forma y color del grano, fueron las más relevantes para caracterizar los genotipos en estudio.

Por otro lado, diferentes análisis indican que el fríjol carauta nombre con el que se le conoce en la Costa Atlántica de Colombia al fríjol lima (P. lunatus L.), tiene 20\% de proteína que, aunque de buen valor biológico, alto contenido de lisina y gran digestibilidad, es deficiente en treonina. Este fríjol posee un glucósido cianogénico, la faseolunatina, y la enzima linamarasa, los cuales se hidrolizan en presencia de humedad en la molienda y liberan glucósidos generando ácido cianhídrico ( $\mathrm{HCN})$, cuyo contenido varía de 10 a más $300 \mathrm{mg} / 100 \mathrm{~g}$ de fríjol. Se acepta con frecuencia que las semillas coloreadas de fríjol lima tienen un alto contenido de glucósidos, aunque algunos estudios reportan ausencia de correlación entre ambos caracteres. Muchos genotipos comerciales tienen sólo de 1 a 8 ppm de HCN en la semilla, en la que generalmente se acepta una concentración límite de 100 a 200 ppm de HCN. Disponible en: https://www.bioversityinternational.org/fileadmin/PGR/article-issue_123-art_4lang_es.html.

De igual manera, Marrugo-Ligardo et al. (2012), informan que realizaron la caracterización del contenido de algunos inhibidores de proteasas, ácido cianhídrico y composición química parcial de la harina integral de tres cultivares, Rojo, Blanco y Moteado, de frijol Zaragoza ( $P$. lunatus L.) de la municipalidad de Túrbaco (Bolívar, Colombia) y refieren que los resultados mostraron algunas diferencias significativas en el contenido de proteína, fibra cruda y ceniza y que el contenido de ácido cianhídrico fue 
menor que las dosis peligrosas para los humanos.

Lo que sirve de referencia para el análisis nutricional y bioquímico de los genotipos de pallar de colores evaluados en el presente estudio, pues no se cuenta con dicha información, teniendo en cuenta que el pallar ( $P$. lunatus L.) es la especie leguminosa de la familia Fabaceae, de particular importancia para la región Ica.

La situación problemática del cultivo de pallar se refiere fundamentalmente a que siendo tan importante para la alimentación humana, por la cantidad y calidad de proteína que aporta a la dieta alimenticia, sobre todo para disminuir los rangos de desnutrición, no está considerado como prioridad en los programas de investigación de las entidades públicas; no se destinan los recursos necesarios para la investigación básica como es el análisis de la diversidad de este importante recurso, que viene estrechando su variabilidad genética, debido a la erosión por las exigencias de uniformidad del mercado preferentemente por el color blanco y en segunda instancia sobre el tamaño del grano; en consecuencia, solamente se cuenta con escasas variedades o cultivares comerciales y cada vez es más difícil encontrar variedades ancestrales de hábito trepador, postrado o semi postrado con diferentes tonalidades en el color, que han sido mantenidos y conservados de generación en generación por las familias de la zona rural.

Se desconoce por tanto la existencia de caracteres o genes importantes para la sanidad vegetal, la nutrición humana, animal o para hacer frente al cambio climático, en este material genético, que no ha sido suficientemente evaluado morfológicamente, agronómicamente y mucho menos molecularmente; por lo tanto, aún no es posible aprovechar dichas bondades en forma directa o incorporándolas al material cultivado a través de programas de mejoramiento genético con hibridaciones dirigidas; por el contrario queda latente el riesgo de erosión genética de este valioso recurso nativo, siendo un problema que la Academia debe estar dispuesta a enfrentar realizando las medidas correctivas oportunas con estrategias de conservación ex situ e in situ, pertinentes, en beneficio de la población.

Como señalan Jacinto et al. (2014), la diversidad de los cultivos a nivel mundial, se encuentra en peligro frecuente debido a cambios constantes, es por eso que la caracterización del germoplasma es fundamental para el conocimiento de la diversidad genética y su aprovechamiento en los programas de fitomejoramiento.

El pallar, es una especie domesticada desde épocas prehispánicas y es una de las 
leguminosas de grano más importantes, habiendo encontrado condiciones agroecológicas excepcionales en los valles de la costa centro sur del Perú y en la región Ica en particular, lo que ha permitido su conservación de generación en generación, destacando por sus cualidades culinarias tanto en consumo de su grano tierno o inmaduro como en grano seco; por lo que se propone hacer frente al riesgo de erosión genética, enviando muestras de semillas al banco de germoplasma del INIA, debiendo promover estrategias de conservación in situ con familias identificadas de la zona rural a fin de garantizar su variabilidad en beneficio de las siguientes generaciones.

La FAO (2018), señala que muchas especies y variedades regionales de alto potencial agrícola se ven desplazadas por otras de origen externo, que presentan generalmente una mayor demanda, capacidad productiva y rentabilidad económica. De esta manera, el rico acervo genético de las variedades locales corre el riesgo de perderse y echar por tierra el esfuerzo ancestral de recolección, selección y desarrollo empírico. Esta situación tiene una serie de efectos como: pérdida de la biodiversidad de las legumbres locales, vulnerabilidad de la seguridad y soberanía alimentaria, decadencia de la economía de la agricultura familiar campesina, dependencia de recursos genéticos externos, entre otros. Todo esto incide directamente en la calidad de vida de los productores. Por lo tanto, es evidente la importancia de la conservación y utilización racional de la variabilidad genética existente, con el fin de preservar los recursos propios. Existe, pues, una profunda necesidad de revalorizar el patrimonio genético de gran riqueza y los conocimientos ancestrales, a veces intangibles y subvalorados.

A través del tiempo, el pallar ha estado en manos de las familias de la zona rural, quienes manteniendo sus costumbres y tradiciones son los grandes conservacionistas de esta especie que nos han transmitido de generación en generación; sin embargo, está corriendo el riesgo de erosión genética, debido a la fuerte presión de los consumidores por determinados tipos, formas y color de granos, en detrimento de la diversidad existente; por lo que no existe en la región Ica, ninguna variedad de pallar de color diferente al blanco que se cultive comercialmente, porque ha sido desplazada por la uniformidad del pallar de grano blanco; por ello, es muy importante, recuperar, identificar, caracterizar y mantener el reservorio de genes de los pallares nativos de colores con estrategias adecuadas de conservación, evitando su erosión genética.

En tal sentido, el presente estudio se realizó con el objetivo de realizar la caracterización 
morfoagronómica, nutricional y evaluación del rendimiento de doce genotipos de pallar de granos de color en condiciones edafoclimáticas de la zona media del valle de Ica.

\section{MATERIALES Y MÉTODOS}

\subsection{Localización del estudio}

El estudio se llevó a cabo en los campos experimentales de la Universidad Nacional "San Luis Gonzaga", ubicado en el caserío Arrabales, distrito Subtanjalla, provincia y departamento de Ica, siendo las coordenadas UTM: 419474.4652 E y 8448986.072 N.

\subsection{Material Biológico}

El material biológico consistió en semilla de doce genotipos de pallar de diverso color, entre los cuales se encuentran tres variedades de grano blanco y blanco cremoso de actual uso comercial, todos de hábito de crecimiento indeterminado. Dicho material fue colectado el año 2018 de diversas localidades de la región Ica, evaluados preliminarmente el año 2019, con la finalidad de incrementar semilla. También se cuenta con un genotipo de pallar de color guinda procedente de la zona andina de la provincia de Cañete y otro de grano bicolor blanco/negro de la costa norte del Perú.

Tabla 1. Genotipos de pallar en estudio

\begin{tabular}{cll}
\hline $\mathbf{N}^{\mathbf{0}}$ & \multicolumn{1}{c}{ Denominación } & \multicolumn{1}{c}{ Detalle original } \\
\hline 1 & PILEM $*-1 / 19$ & Grano arriñonado blanco SP, tipo Generoso \\
2 & PILEM-4/19 T & Grano arriñonado blanco, tipo Generoso grande \\
3 & PILEM-5/19 & Grano cuadrado blanco cremoso SP \\
4 & PILEM-7/19 & Grano arriñonado, variegado marfil/guinda \\
5 & PILEM-8/19 & Grano arriñonado, sombreado cenizo oscuro \\
6 & PILEM-18/19 & Grano arriñonado, bicolor: blanco/negro \\
7 & PILEM-18-1/19 & Grano arriñonado, mancha basal negro \\
8 & PILEM 20/19 & Grano cilíndrico guinda oscuro \\
9 & PILEM-22/19 & Grano arriñonado variegado maculado/granate \\
10 & PILEM-23/19 & Grano arriñonado rosado com chispitas oscuras \\
11 & PILEM-24/19 & Grano arriñonado, mancha basal negro \\
12 & PILEM-25/19 & Grano arriñonado, mancha basal guinda \\
\hline
\end{tabular}

\subsection{Siembra y conducción del campo experimental}

El terreno donde se realizó el estudio, se caracteriza por ser de textura franco arenoso, de reacción moderadamente alcalina, con bajo contenido de nitrógeno y materia orgánica, alto contenido de fósforo, contenido medio de carbonato de calcio, muy ligeramente 
salino, baja capacidad de intercambio catiónico y contenido alto de calcio y bajo en los cationes cambiables sodio y potasio.

La semilla previamente desinfectada con Rhizolex $\mathrm{T}$ y Vencetho a razón de $4 \mathrm{~g} / \mathrm{kg}$ de semilla, se sembró el 16 de abril, colocando tres semillas por sitio en la costilla del surco, a un distanciamiento de 1,5 m entre golpes, según el croquis experimental. Sólo fue necesario resembrar el tratamiento 8(PILEM 20/19), por su bajo porcentaje de emergencia, 15 días después se realizó la única fertilización al suelo, empleando la fórmula 40-60-40 de $\mathrm{N}-\mathrm{P}_{2} \mathrm{O}_{5}-\mathrm{K}_{2} \mathrm{O} \mathrm{kg} \mathrm{ha}{ }^{-1}$ con urea, fosfato di amónico y sulfato de potasio, respectivamente, cuya mezcla se aplicó de manera dirigida en cada sitio de plantas. Se aplicaron doce riegos por gravedad, siendo ligeros con una frecuencia de 15 días aproximadamente. Se mantuvo el campo libre de malezas y la plaga que se presentó con mayor frecuencia fue "cigarrita verde" (Empoasca kraemeri) que afectó el área foliar de la planta, disminuyendo su capacidad fotosintética. Dada la diferencia en el ciclo de los genotipos en evaluación, la cosecha fue escalonada, desde los 168 días para los que mostraron cierta precocidad, hasta los 188 días para los tardíos; de igual manera, la trilla se realizó de manera escalonada, evaluando las dimensiones y variables cualitativas de la vaina, dimensiones del grano y el peso de grano por planta.

\subsection{Caracterización morfológica y morfoagronómica}

Se seleccionaron descriptores genéticos del Instituto Internacional para los Recursos Genéticos Vegetales (IPGRI, 2001), para Phaseolus lunatus L., tanto para variables cualitativas como cuantitativas: color de cotiledones $(\mathrm{CC})$, color del hipocotilo $(\mathrm{CH})$, color de la nervadura de las hojas primarias (CNHP), antocianina en las hojas (AH), pubescencia en las hojas (PH), color del estandarte (CE), color de la quilla (CQ), color de las alas (CA), curvatura de la vaina (CV), forma del ápice de la vaina (FAV), forma de la semilla (FS), color del fondo de la semilla (CFS), patrón del tegumento de la semilla (PTS), días al 50\% de floración (DF50\%), días a la madurez de la vaina (DMV), número de vainas por planta (NVP), longitud de la guía principal (LGP), cobertura de follaje (CF), largo de vaina (LV), ancho de vaina (AV), largo de grano (LG), ancho de grano (AG), grosor del grano (GG), número de granos por vaina (NGV), peso de 100 semillas (P100S), peso de grano por planta $(\mathrm{g})$.

\subsection{Evaluación del rendimiento}

La evaluación comparativa del rendimiento y sus principales componentes agronómicos, 
se realizó a través del Diseño en Bloques Completos al Azar (DBCA), los análisis de varianza (ANVA) se realizaron a través de la prueba de significación de F, al nivel 0,05 de probabilidad y para la comparación de promedios se utilizó la Prueba de Rango Múltiple de Duncan al 0,05. Para medir el grado de relación de las variables cuantitativas se utilizó el Coeficiente de Correlación de Pearson (Milton, 2007).

\section{RESULTADOS Y DISCUSIÓN}

En la tabla 2, se presentan los cuadrados medios de los análisis de varianza realizados a las principales variables cuantitativas de los genotipos de pallar en estudio, donde se observa que para cobertura de follaje (CF) se encontró diferencia significativa entre los tratamientos y diferencia altamente significativa entre repeticiones, con un coeficiente de variación de 19,27\%, de manera similar, para el número de vainas por planta (NVP), se ha encontrado diferencia significativa entre tratamientos, más no así entre repeticiones, con un coeficiente de variación de $19,01 \%$.

Se ha encontrado diferencia altamente significativa entre tratamientos para longitud de la guía principal (LGP), días al 50\% de floración (DF50\%), número de granos por vaina (NGV), peso de 100 granos (P100G), días a madurez de la vaina (DMV) y peso de grano por planta (PGP), sin diferencia significativa para las repeticiones, con excepción de la diferencia altamente significativa entre las repeticiones para los días a la madurez de la vaina y la diferencia significativa entre repeticiones para el peso de granos por planta, con coeficientes de variación de 19,$27 ; 10,56 ; 1,72 ; 19,01 ; 6,14 ; 7,79 ; 0,63$ y 13,34\%, respectivamente para cada una de las variables mencionadas.

Tabla 2 Cuadrados medios de los Análisis de varianza de variables morfoagronómicas de doce genotipos de pallar de granos de colores

\begin{tabular}{|c|c|c|c|c|c|c|c|c|c|c|c|}
\hline \multirow[b]{2}{*}{$\begin{array}{l}\text { Fuentes de } \\
\text { variación }\end{array}$} & \multirow[b]{2}{*}{ G.L. } & \multirow[b]{2}{*}{$\begin{array}{c}\text { Cobertura de } \\
\text { follaje }\end{array}$} & \multirow[b]{2}{*}{$\begin{array}{l}\text { Longitud guía } \\
\text { principal }\end{array}$} & \multirow[b]{2}{*}{$\begin{array}{c}\text { Días al } \\
50 \% \\
\text { Floración } \\
\end{array}$} & \multicolumn{3}{|c|}{ Cuadrados medios } & \multirow[b]{2}{*}{$\begin{array}{c}\text { Día a } \\
\text { madurez } \\
\text { de vaina }\end{array}$} & \multirow[b]{2}{*}{$\begin{array}{c}\text { Peso de } \\
\text { grano por } \\
\text { planta } \\
\end{array}$} & \multicolumn{2}{|c|}{$\mathrm{Ft}$} \\
\hline & & & & & $\begin{array}{l}\text { Número } \\
\text { Vainas/ } \\
\text { planta } \\
\end{array}$ & $\begin{array}{c}\text { Número de } \\
\text { granos por } \\
\text { vaina } \\
\end{array}$ & $\begin{array}{c}\text { Peso de } 100 \\
\text { Granos }\end{array}$ & & & 0,05 & 0,01 \\
\hline Tratamientos & 11 & $9832045.474 *$ & $2083.619 * *$ & $146.181 * *$ & $261.085 *$ & $0.271 * *$ & $4812.290 * *$ & $119.967 *$ & $12491.705 *$ & 2.259 & 3.184 \\
\hline Repeticiones & 2 & $68401287.111 *$ & $718.609 \mathrm{NS}$ & $5.583 \mathrm{NS}$ & $27.710 \mathrm{NS}$ & $0.067 \mathrm{NS}$ & $144.210 \mathrm{NS}$ & $17.027 * *$ & $3190.775 *$ & 3.443 & 5.719 \\
\hline Error & 22 & 3915731.535 & 270.798 & 1.765 & 92.479 & 0.025 & 142.528 & 1.270 & 808.180 & & \\
\hline Total & 35 & & & & & & & & & & \\
\hline $\begin{array}{c}\text { Coeficiente } \\
\text { de Variación } \\
(\%)\end{array}$ & & 19.27 & 10.56 & 1.72 & 19.01 & 6.14 & 7.79 & 0.63 & 13.34 & & \\
\hline
\end{tabular}

NS.- No existe diferencia significativa

*.- Existe diferencia significativa (95\% de confiabilidad)

**.- Existe diferencia altamente significativa (99\% de confiabilidad) 
En la tabla 3, se observa que existe diferencia altamente significativa entre los tratamientos para largo de vaina (LV), ancho de vaina (AV), largo de grano (LG) y ancho de grano (AG), diferencia significativa entre tratamientos para el grosor del grano (GG), con coeficientes de variación entre 6,$02 ; 8,46 ; 5,74 ; 7,09$ y 4,91\%, respectivamente.

Tabla 3. Cuadrados medios de los Análisis de varianza de las dimensiones de vainas y granos de doce genotipos de pallar de granos de colores

\begin{tabular}{lcccccccc}
\hline \multirow{2}{*}{$\begin{array}{c}\text { Fuentes de } \\
\text { variación }\end{array}$} & G.L. & \multicolumn{9}{c}{$\begin{array}{c}\text { Largo } \\
\text { de vaina }\end{array}$} & $\begin{array}{c}\text { Ancho } \\
\text { de vaina }\end{array}$ & $\begin{array}{c}\text { Largo de } \\
\text { grano }\end{array}$ & $\begin{array}{c}\text { Ancho } \\
\text { de grano }\end{array}$ & $\begin{array}{c}\text { Grosor del } \\
\text { grano }\end{array}$ & $\mathbf{0 , 0 5}$ & $\mathbf{0 , 0 1}$ \\
\hline Tratamientos & 11 & $7.169^{* *}$ & $0.271^{* *}$ & $0.291^{* *}$ & $0.088^{* *}$ & $0.0021^{*}$ & 2.259 & 3.184 \\
Repeticiones & 2 & $0.680 \mathrm{NS}$ & $0.092 *$ & $0.044 \mathrm{NS}$ & $0.077 * *$ & $0.0019 \mathrm{NS}$ & 3.443 & 5.719 \\
$\begin{array}{l}\text { Error } \\
\text { Total }\end{array}$ & 22 & 0.3604 & 0.025 & 0.0193 & 0.0104 & 0.0009 & & \\
\hline \multicolumn{1}{c}{ C. V. (\%) } & 35 & & 6.02 & 8.46 & 5.74 & 7.09 & 4.91 & \\
\hline
\end{tabular}

NS.- No existe diferencia significativa

*.- Existe diferencia significativa (95\% de confiabilidad)

**.- Existe diferencia altamente significativa ( $99 \%$ de confiabilidad)

En la tabla 4, de la prueba de rango múltiple de Duncan, realizada para las variables evaluadas se observa que, en cobertura de follaje, casi todos los genotipos de pallar mostraron escasa diferencia entre ellos, con un rango de $12318 \mathrm{~cm}^{2}$ de cobertura de follaje para PILEM 7/19, de grano variegado, hasta $9913.33 \mathrm{~cm}^{2}$ para PILEM 1/19 de grano blanco; en la longitud de la guía principal, tres genotipos se ubicaron en el primer lugar con valores entre 203,67; 191,78 y $188,67 \mathrm{~cm}$ de longitud, seguidos de seis genotipos que se ubicaron en el segundo y tercer lugar con valores entre 170,22 hasta $139,89 \mathrm{~cm}$; finalmente tres genotipos se ubicaron en el cuarto lugar con 135,33; 127,78 y $125 \mathrm{~cm}$ de longitud de guía, siendo las más cortas del estudio.

Tabla 4. Prueba de Rango Múltiple de Duncan de las variables morfoagronómicas de doce genotipos de pallar de granos de colores

\begin{tabular}{|c|c|c|c|c|c|c|c|c|c|c|c|c|c|c|c|c|}
\hline \multirow{2}{*}{$\begin{array}{l}\text { Geno } \\
\text { tipos }\end{array}$} & \multicolumn{2}{|c|}{$\mathrm{CF}$} & \multicolumn{2}{|c|}{ LGP } & \multicolumn{2}{|c|}{ DF $50 \%$} & \multicolumn{2}{|c|}{ NVP } & \multicolumn{2}{|c|}{ NGV } & \multicolumn{2}{|c|}{ P100G } & \multicolumn{2}{|c|}{ DMV } & \multicolumn{2}{|c|}{ PGP } \\
\hline & $\begin{array}{l}\text { Prom. } \\
(\mathrm{cm} 2)\end{array}$ & $\begin{array}{c}\text { Duncan } \\
0.05\end{array}$ & $\begin{array}{l}\text { Prom. } \\
(\mathrm{cm})\end{array}$ & $\begin{array}{c}\text { Duncan } \\
0.05\end{array}$ & $\begin{array}{l}\text { Prom. } \\
\text { (días) }\end{array}$ & $\begin{array}{c}\text { Duncan } \\
0.05\end{array}$ & $\begin{array}{l}\text { Prom. } \\
\text { (unid) }\end{array}$ & $\begin{array}{c}\text { Duncan } \\
0.05\end{array}$ & $\begin{array}{l}\text { Prom. } \\
\text { (unid) }\end{array}$ & $\begin{array}{c}\text { Duncan } \\
0.05\end{array}$ & $\begin{array}{l}\text { Prom. } \\
\text { (g) }\end{array}$ & $\begin{array}{c}\text { Duncan } \\
0.05\end{array}$ & $\begin{array}{l}\text { Prom. } \\
\text { (días) }\end{array}$ & $\begin{array}{c}\text { Duncan } \\
0.05\end{array}$ & $\begin{array}{l}\text { Prom. } \\
(\mathrm{g})\end{array}$ & $\begin{array}{c}\text { Duncan } \\
0.05\end{array}$ \\
\hline 1 & 9413.3 & & 188.67 & $a b$ & 67.33 & $a b$ & 55.23 & a & 2.37 & def & 181 & $a b$ & 169.33 & $a b$ & 244.90 & $a b c$ \\
\hline 2 & 10736.67 & a & 170.22 & $\mathrm{bc}$ & 82.00 & ef & 49.40 & a & 2.33 & ef & 179. & $a b c$ & 183.00 & fg & 225.59 & $\mathrm{bc}$ \\
\hline 3 & 10841.33 & $\mathrm{a}$ & 139.89 & $\mathrm{~cd}$ & 79.00 & $\mathrm{e}$ & 46.78 & a & 3.25 & a & 194.71 & $\mathrm{a}$ & 180.00 & $\mathrm{e}$ & 294.85 & a \\
\hline 4 & 12318.00 & a & 143.33 & $\mathrm{~cd}$ & 82.33 & $\mathrm{f}$ & 63.75 & a & 2.42 & cdef & 175.91 & $a b c$ & 184.33 & & 282.49 & $a b$ \\
\hline 5 & 9956.67 & a & & $\mathrm{d}$ & & ef & & a & & $\mathrm{bc}$ & 186.95 & a & & d & & abcd \\
\hline 6 & 9873.33 & a & 191.78 & $\mathrm{ab}$ & 76.67 & bcd & 48.32 & $\mathrm{a}$ & 2.79 & $\mathrm{~b}$ & 102.89 & e & 181.67 & ef & 164.75 & $\mathrm{e}$ \\
\hline 7 & 11243.00 & $\mathrm{a}$ & 203.67 & $\mathrm{a}$ & 82.33 & f & 49.57 & a & 2.83 & b & 157.33 & $\mathrm{~cd}$ & 174.67 & c & 234.99 & bcd \\
\hline 8 & 5393.33 & $b$ & 125.00 & d & 86.33 & & 27.00 & $\mathrm{~b}$ & 2.59 & bcde & 71.47 & $\mathrm{f}$ & 188.67 & $\mathrm{r}$ & 56.79 & $\mathrm{f}$ \\
\hline 9 & 12302.00 & & 144.67 & $\mathrm{~cd}$ & 84.33 & f & 54.91 & a & 2.33 & ef & 181.76 & $a b$ & 182.33 & & 253.99 & $a b c$ \\
\hline 10 & 11152.00 & $\mathrm{a}$ & 148.00 & $\mathrm{~cd}$ & & 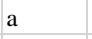 & & a & 2.24 & $\mathrm{f}$ & 141.80 & d & 171.33 & $\mathrm{~b}$ & 213.99 & cde \\
\hline 11 & 9477.00 & & 127.78 & $\mathrm{~d}$ & 76.33 & $\mathrm{bc}$ & 54.01 & a & 2.67 & bcd & 103.08 & $\mathrm{e}$ & 177.00 & d & 161.18 & $\mathrm{e}$ \\
\hline 12 & 10512.00 & & 151.33 & $\mathrm{~cd}$ & 67.67 & $a b$ & 50.77 & $\mathrm{a}$ & 2.27 & $\mathrm{f}$ & 162.44 & bcd & 168.33 & $\mathrm{a}$ & 184.04 & de \\
\hline
\end{tabular}

Nota.- Los genotipos que muestran la misma letra, no son significativamente diferentes entre sí. 
En los días al inicio de la floración (50\%), tres genotipos iniciaron su floración antes de los 70 días, siendo percibidos como los más precoces, siendo de hábito de crecimiento indeterminado, cuatro genotipos iniciaron su floración antes de los 80 días, lo que coincide con las variedades de actual uso comercial, y, cinco genotipos iniciaron su floración entre los 82 a 86 días, siendo los más tardíos. En el número de vainas por planta (NVP), solamente el genotipo 8 (PILEM 20/19), presentó el menor valor con 27 vainas por planta, ubicándose en el segundo lugar; todos los demás genotipos presentaron valores similares, desde 63,75 a 45,43 vainas por planta (tabla 4).

En cuanto al número de granos por vaina, destaca el genotipo 3 (PILEM 5/19) con 3,25 granos por vaina, en promedio, ubicándose en el primer lugar; seguido de cinco genotipos que se ubicaron en el segundo lugar con valores entre 2,83 y 2,59 granos por vaina, en promedio; los menores valores fueron para los genotipos de clave 1, 2 12, 9 y 10 con 2,37 a 2,24 granos por vaina, en promedio; en el peso de 100 granos, seis genotipos se ubicaron en el primer lugar con valores entre 194,71 g para el genotipo 3(PILEM 5/19), hasta 175,91 g para 4(PILEM 7/19); el menor valor fue para el genotipo 8(PILEM 20/19) con 71.47 g en 100 granos; en los días a la madurez de la vaina, destacaron en el primer como los más precoces, los genotipos 12(PILEM 25/19) y 1(PILEM 1/19) con 168,33 y 169,33 días a la madurez de la vaina; cuatro genotipos alcanzaron la madurez de la vaina entre los 171,33 y 177,67 días; seis genotipos alcanzaron la madurez de la vaina entre los 180 y 188,67 días, siendo el más tardío 8(PILEM 20/19), de procedencia de la zona andina de la provincia de Cañete; en el peso de granos por planta, siete genotipos lograron los mayores rendimientos con pesos de $225,59 \mathrm{~g} /$ planta, equivalente a $2005,27 \mathrm{~kg} \mathrm{ha}^{-1}$ para el genotipo 2(PILEM 4/19), testigo de grano blanco, hasta 294,85 g/planta equivalente a 2 620,86 kg ha-1 para el genotipo 3(PILEM 5/19); cuatro genotipos obtuvieron rendimientos por debajo de las 2 ton $\mathrm{ha}^{-1}$, desde 213,99 g/planta, equivalente a 1902,18 $\mathrm{kg} \mathrm{ha}^{-1}$ para el genotipo 10(PILEM 23/19), hasta 161,18 g/planta, equivalente a 1432,69 $\mathrm{kg} \mathrm{ha}^{-1}$ para el genotipo 11(PILEM 24/19). De manera excepcional, es de mencionar al genotipo 8(PILEM 20/19) que fue el más tardío, de escasa adaptación, que alcanzó el más bajo peso con 56,79 g/planta equivalente a solamente $504,84 \mathrm{~kg} \mathrm{ha}^{-1}$ de grano (tabla 4). En lo que respecta a las dimensiones de la vaina, el genotipo 5(PILEM 8/19) alcanzó el mayor largo de vaina con $11,78 \mathrm{~cm}$, ubicándose en el primer lugar, junto con otros tres genotipos, hasta 11,07 cm del genotipo 7(PILEM 18-1/19); los genotipos 6, 11 y 12 se 
ubicaron en el segundo, tercer y cuarto lugar con 10,32; 10,11 y 10,02 cm de largo de vaina; cuatro genotipos alcanzaron largo de vaina entre 8 y $10 \mathrm{~cm}$; finalmente el genotipo 8(PILEM 20/19), ocupó el último lugar con un largo de vaina de 5,99 cm, siendo el valor más bajo correspondiente al genotipo procedente de la zona andina de Cañete (tabla 5).

Tabla 5. Prueba de Rango Múltiple de Duncan de las variables morfoagronómicas de doce genotipos de pallar de granos de colores

\begin{tabular}{ccccccccccc}
\hline \multirow{2}{*}{$\begin{array}{c}\text { Geno } \\
\text { tipos }\end{array}$} & \multicolumn{3}{c}{ LV } & \multicolumn{2}{c}{ AV } & \multicolumn{2}{c}{ LG } & \multicolumn{2}{c}{ AG } & \multicolumn{2}{c}{ GG } \\
& $\begin{array}{c}\text { (cm) } \\
\text { (cm }\end{array}$ & $\begin{array}{c}\text { Duncan } \\
\mathbf{0 . 0 5}\end{array}$ & $\begin{array}{c}\text { Prom } \\
(\mathbf{c m})\end{array}$ & $\begin{array}{c}\text { Duncan } \\
\mathbf{0 . 0 5}\end{array}$ & $\begin{array}{c}\text { Prom. } \\
(\mathbf{c m})\end{array}$ & $\begin{array}{c}\text { Duncan } \\
\mathbf{0 . 0 5}\end{array}$ & $\begin{array}{c}\text { Prom } \\
\text { (unid) }\end{array}$ & $\begin{array}{c}\text { Duncan } \\
\mathbf{0 . 0 5}\end{array}$ & $\begin{array}{c}\text { Prom } \\
\text { (cm) }\end{array}$ & $\begin{array}{c}\text { Duncan } \\
\mathbf{0 . 0 5}\end{array}$ \\
\hline 1 & 11.24 & $\mathrm{abc}$ & 2.42 & $\mathrm{a}$ & 2.63 & $\mathrm{ab}$ & 1.53 & $\mathrm{~b}$ & 0.63 & $\mathrm{abc}$ \\
2 & 9.85 & $\mathrm{e}$ & 1.83 & $\mathrm{~cd}$ & 2.66 & $\mathrm{a}$ & 1.49 & $\mathrm{bcd}$ & 0.62 & $\mathrm{abcd}$ \\
3 & 11.35 & $\mathrm{ab}$ & 2.33 & $\mathrm{ab}$ & 2.37 & $\mathrm{bcde}$ & 1.78 & $\mathrm{a}$ & 0.65 & $\mathrm{ab}$ \\
4 & 9.74 & $\mathrm{e}$ & 1.94 & $\mathrm{~cd}$ & 2.76 & $\mathrm{a}$ & 1.48 & $\mathrm{bcd}$ & 0.62 & $\mathrm{abcd}$ \\
5 & 11.78 & $\mathrm{a}$ & 1.99 & $\mathrm{c}$ & 2.61 & $\mathrm{ab}$ & 1.52 & $\mathrm{bc}$ & 0.61 & $\mathrm{bcd}$ \\
6 & 10.32 & $\mathrm{bcde}$ & 1.66 & $\mathrm{~d}$ & 2.21 & $\mathrm{e}$ & 1.32 & $\mathrm{~cd}$ & 0.61 & $\mathrm{bcd}$ \\
7 & 11.07 & $\mathrm{abcd}$ & 1.83 & $\mathrm{~cd}$ & 2.38 & $\mathrm{bcde}$ & 1.41 & $\mathrm{bcd}$ & 0.61 & $\mathrm{bcd}$ \\
8 & 5.99 & $\mathrm{~g}$ & 1.30 & $\mathrm{e}$ & 1.58 & $\mathrm{f}$ & 1.07 & $\mathrm{e}$ & 0.68 & $\mathrm{a}$ \\
9 & 9.62 & $\mathrm{e}$ & 1.75 & $\mathrm{~cd}$ & 2.65 & $\mathrm{a}$ & 1.47 & $\mathrm{bcd}$ & 0.61 & $\mathrm{bcd}$ \\
10 & 8.52 & $\mathrm{f}$ & 1.81 & $\mathrm{~cd}$ & 2.34 & $\mathrm{de}$ & 1.37 & $\mathrm{bcd}$ & 0.63 & $\mathrm{abc}$ \\
11 & 10.11 & $\mathrm{cde}$ & 1.66 & $\mathrm{~d}$ & 2.35 & $\mathrm{cde}$ & 1.29 & $\mathrm{~d}$ & 0.57 & $\mathrm{e}$ \\
12 & 10.02 & $\mathrm{de}$ & 2.04 & $\mathrm{bc}$ & 2.50 & $\mathrm{abcd}$ & 1.52 & $\mathrm{~b}$ & 0.62 & $\mathrm{abcd}$ \\
\hline
\end{tabular}

Nota.- Los genotipos que muestran la misma letra, no son significativamente diferentes entre sí.

En el ancho de vaina, los genotipos 1(PILEM 1/19) y 3(PILEM 5/19) destacan en el primer lugar con 2,42 y 2,33 cm en promedio, respectivamente; seguidos del genotipo 12 que también presentó un ancho de vaina de 2,04 cm; seguidamente, seis genotipos se ubicaron en el tercer lugar con valores entre 1,99 cm para 5(PILEM 8/19) y 1,75 cm para 9(PILEM 22/19), de ancho de vaina; los genotipos 11 y 6 obtuvieron 1,66 cm de ancho de vaina cada uno, y el genotipo 8(PILEM 20/19) presentó 1,30 cm de ancho de vaina, siendo significativamente diferente e inferior a los demás genotipos evaluados (tabla 5). Los genotipos que se ubicaron en el primer lugar con el mayor largo del grano fueron seis, desde 4(PILEM 7/19) con $2.76 \mathrm{~cm}$ hasta el genotipo 12(PILEM 25/19) con 2,50 cm de largo de grano; cuatro genotipos se ubicaron en el segundo, tercer y cuarto lugar con valores entre 2,38 y 2,34 cm de largo de grano; el genotipo 6(PILEM 18/19) obtuvo 2,21 cm de largo de grano y se ubicó en el quinto lugar, y el genotipo 8(PILEM 20/19) se ubicó en el último lugar con 1,58 cm de largo de grano (tabla 5).

En el ancho de grano, destaca en el primer lugar solamente el genotipo 3(PILEM 5/19) con 1,78 cm; en el segundo lugar se ubicaron ocho genotipos, desde 1 (PILEM 1/19) con 
1,53 cm, hasta 10(PILEM 24/19) con $1.37 \mathrm{~cm}$ de ancho de grano; seguidamente, en los últimos lugares se ubicaron 6 (PILEM 18/19), 11(PILEM 24/19 y 8(PILEM 20/19) con $1.32,1.29$ y $1.07 \mathrm{~cm}$ de ancho de grano, respectivamente (tabla 5).

En el grosor del grano, siete genotipos se ubicaron en el primer lugar, destacando 8(PILEM 20/19) con 0,68 cm, hasta 2(PILEM 4/19) con 0,62 cm de grosor del grano; en segundo lugar se ubicaron los genotipos $5,7,9$ y 6 con $0.61 \mathrm{~cm}$ de grosor del grano, quedando al final el genotipo 11 (PILEM 24/19) con 0,57 cm de grosor del grano, siendo el genotipo de granos más aplanados (tabla 5).

Tabla 6. Coeficientes de correlación entre 13 variables cuantitativas de la caracterización morfoagronómica de doce genotipos de pallar de granos de colores

\begin{tabular}{|c|c|c|c|c|c|c|c|c|c|c|c|c|c|}
\hline & $\mathrm{CF}$ & LGP & DF50\% & NVP & NGV & P100G & DMV & PGP & LV & $\mathrm{AV}$ & LG & $\mathrm{AG}$ & GG \\
\hline LGP & 0.23727 & 1 & & & & & & & & & & & \\
\hline DF50\% & o -0.1054 & -0.1688 & 1 & & & & & & & & & & \\
\hline NVP & 0.8228 **: & 0.18525 & -0.44435 & 1 & & & & & & & & & \\
\hline NGV & -0.1296 & 0.01695 & 0.330789 & -0.37818 & 1 & & & & & & & & \\
\hline P100G & $0.7024 *$ & 0.14775 & -0.11145 & 0.46967 & -0.0345 & 1 & & & & & & & \\
\hline DMV & -0.249 & -0.3262 & $0.8737 * *$ & -0.4421 & 0.23704 & -0.28996 & 1 & & & & & & \\
\hline PGP & 0.8405 *⿻丷木 & 0.20167 & -0.08387 & $0.6613 *$ & 0.11895 & $0.9098 * *$ & -0.2299 & 1 & & & & & \\
\hline LV & 0.54767 & 0.40813 & -0.2318 & 0.39657 & 0.36767 & $0.6729 *$ & -0.4479 & $0.7182 * *$ & 1 & & & & \\
\hline $\mathrm{AV}$ & 0.43907 & 0.24825 & -0.48797 & 0.43031 & 0.13381 & $0.7976 * *$ & -0.571 & $0.7603 * *$ & 0.7422 ** & 1 & & & \\
\hline LGP & $0.8275 * \because$ & 0.21043 & -0.20881 & $0.7649 * *$ & -0.2779 & $0.8358 * *$ & -0.3401 & $0.8353 * *$ & $0.6936 *$ & 0.6377 **: & & & \\
\hline AG & $0.6307 *$ & 0.11007 & -0.22459 & 0.40388 & 0.24874 & $0.8975 * *$ & -0.3362 & $0.8695 * *$ & $0.7488 * \because$ & 0.8879 ** & $0.6969 *$ & 1 & \\
\hline GG & -0.4973 & -0.2022 & 0.112833 & -0.52972 & 0.07814 & -0.10118 & 0.26243 & -0.23243 & -0.5326 & -0.0066 & -0.5388 & -0.0706 & 1 \\
\hline
\end{tabular}

*.- Significación al 0.05 de probabilidad

**.- Significación al 0.01 de probabilidad

En la tabla 6, se presentan los coeficientes de correlación entre 13 variables cuantitativas evaluadas en doce genotipos de pallar de crecimiento indeterminado y de granos de colores diversos, resaltando la alta correlación positiva existente $(>0,708)$ entre el número de vainas por planta (NVP) con la cobertura de follaje (CF); los días a la madurez de la vaina (DMV) con los días a la floración (DF50\%); el peso de granos por planta (PGP) con longitud de la guía principal (LGP); el largo de vaina (LV) con el peso de grano por planta (PGP); el ancho de vaina (AV) con el peso de 100 granos (P100G), peso de granos por planta y largo de vaina; la longitud de la guía principal con la cobertura de follaje, número de vainas por planta, el peso de 100 granos, peso de grano por planta y ancho de la vaina; el ancho de grano (AG) con el peso de 100 granos, peso de grano por 
planta, largo y ancho de vaina.

Se ha encontrado correlación positiva significativa (>0,576) entre el peso de 100 granos y la cobertura del follaje; entre el peso de grano por planta con el número de vainas por planta; entre el largo de vaina con el peso de 100 granos; entre el ancho de grano, la cobertura de follaje y el largo del grano. No se ha encontrado correlación alguna con el grosor del grano (GG) (tabla 6).

En la tabla 7, se presenta un resumen de las variables cualitativas evaluadas a los doce genotipos de pallar, habiéndose considerado la alternativa de mayor frecuencia en las plantas muestreadas.

Tabla 7. Variables cualitativas evaluadas a doce genotipos de pallar de granos de colores

\begin{tabular}{|c|c|c|c|c|c|c|c|c|c|c|c|c|c|c|}
\hline Clave & Genotipos & $\mathrm{CC}$ & $\mathrm{CH}$ & CNHP & $\mathrm{AH}$ & PH & $\mathrm{CE}$ & $\mathrm{CQ}$ & $\mathrm{CA}$ & $\mathrm{CV}$ & FAV & FS & CFS & PTS \\
\hline 1 & PILEM*-1/19 & Verde & Verde & Verde & Ausente & Glabra & Violeta & Verde & Violeta & Lig. Curva & Medio & Arriñonada & Blanco & $\begin{array}{l}\text { Ausencia } \\
\text { de mácula }\end{array}$ \\
\hline 2 & PILEM-4/19 T & Verde & Verde & Verde & Ausente & Glabra & Blanco & Verde & Blanco & Curva & Medio & Arriñonada & Blanco & $\begin{array}{l}\text { Ausencia } \\
\text { de mácula }\end{array}$ \\
\hline 3 & PILEM-5/19 & Verde & Verde & Verde & Ausente & Glabra & Blanco & Verde & Blanco & Recta & Corto & Cuadrada & Blanco & $\begin{array}{l}\text { Ausencia } \\
\text { de mácula }\end{array}$ \\
\hline 4 & PILEM-7/19 & Verde & Verde & Verde & Ausente & Glabra & Blanco & Verde & Blanco & Curva & Medio & Arriñonada & Crema & Maculada \\
\hline 5 & PILEM-8/19 & Verde & Verde & Verde & Ausente & Glabra & Violeta & Verde & Violeta & Lig. Curva & Largo & Arriñonada & Cenizo & $\begin{array}{l}\text { Manchas } \\
\text { dispersas }\end{array}$ \\
\hline 6 & PILEM-18/19 & Verde & Verde & Verde & Ausente & Glabra & Violeta & Púrpura & Púrpura & Lig. Curva & Largo & Arriñonada & $\begin{array}{c}\text { Blanco/ } \\
\text { negro }\end{array}$ & $\begin{array}{c}\text { Hillium } \\
\text { maculado }\end{array}$ \\
\hline 7 & PILEM-18-1/19 & Verde & Verde & Verde & Ausente & Glabra & Violeta & Verde & Violeta & Lig. Curva & Largo & Arriñonada & Crema & $\begin{array}{c}\text { Mancha } \\
\text { basal }\end{array}$ \\
\hline 8 & PILEM 20/19 & Verde & Verde & Verde & Ausente & Glabra & Blanco & Verde & Blanco & Lig. Curva & Corto & Cilindrica & Guinda & $\begin{array}{l}\text { Ausencia } \\
\text { de mácula }\end{array}$ \\
\hline 9 & PILEM-22/19 & Verde & Verde & Verde & Ausente & Glabra & Blanco & Verde & Rosa claro & Curva & Largo & Arriñonada & Crema & Maculada \\
\hline 10 & PILEM-23/19 & Verde & Verde & Verde & Ausente & Glabra & Rosa claro & Verde & Rosa claro & Curva & Medio & Elíptica & Marfil & $\begin{array}{l}\text { Manchas } \\
\text { dispersas }\end{array}$ \\
\hline 11 & PILEM-24/19 & Verde & Verde & Verde & Ausente & Glabra & Violeta & Verde & Violeta & Lig. Curva & Largo & Rectangular & $\begin{array}{l}\text { Blanco/ } \\
\text { negro }\end{array}$ & $\begin{array}{c}\text { Mancha } \\
\text { basal }\end{array}$ \\
\hline 12 & PILEM-25/19 & Verde & Verde & Verde & Ausente & Glabra & Blanco & Verde & Blanco & Lig. Curva & Medio & Arriñonada & Marfil & $\begin{array}{c}\text { Mancha } \\
\text { basal }\end{array}$ \\
\hline
\end{tabular}

$\mathrm{CC}$ : color de cotiledones, $\mathrm{CH}$ : color de hipocotilo, CNHP: color de nervadura en hojas primarias, $\mathrm{AH}$ : antocianinas en hojas, $\mathrm{PH}$ : pubescencia en hojas, $\mathrm{CE}$ : color estandarte, CQ: color quilla, CA: color alas, CV: curvatura vaina, FAV: forma del ápice de vaina, FS: forma de semilla, CFS: color de fondo de semilla, PTS: patrón de tegumento de semilla.

Los genotipos de pallar evaluados, presentan similitud en variables como color de cotiledones, color del hipocótilo, color de la nervadura principal, siendo el 100\% de color verde; ninguno presenta antocianinas en las hojas, todos presentan hojas glabras, sin pubescencia. Hay variación en la coloración de estandarte, quilla y alas de las flores de los genotipos evaluados, lo que define el color de las flores. Las mayoría de genotipos 
presentaron vainas curvas y ligeramente curvas, también un genotipo presentó vainas rectas o casi rectas; el ápice de las vainas fueron $40 \%$ largas, $40 \%$ medias y $20 \%$ cortas. Los granos presentaron colores variados desde blanco, crema, marfil, maculados, guinda, combinados blanco con negro, variegados crema con guinda, gris, etc., mostrando una tendencia a la segregación en genotipos que seguramente tienen un mayor porcentaje de cruzamiento natural que el esperado en leguminosas autógamas.

Con el interés de conocer las características morfoagronómicas relevantes de un material genético que se ha venido transmitiendo de manera limitada de generación en generación por los agricultores tradicionales y dadas las exigencias de uniformidad en el color, la forma del grano, precocidad, hábito de crecimiento determinado, se ha ido reduciendo peligrosamente la diversidad del pallar, recurso genético nativo y muy arraigado en la región Ica; por lo que la evaluación preliminar realizada en el presente estudio ha permitido identificar lo siguiente:

Existe diferencia significativa y altamente significativa entre la mayoría de las variables evaluadas, con preferencia en las variables cuantitativas que están vinculadas o correlacionadas con la producción, lo que estaría indicando que se cuenta con una importante variabilidad genética que es importante caracterizar, evaluar, mantener y conservar en condiciones ex situ y fomentar la conservación in situ, utilizando estrategias pertinentes con pobladores de la zona rural; quienes luego de conocer su comportamiento, puedan cultivar este material genético para autoconsumo e intercambio con otras zonas rurales y darle importancia al mantenimiento de este recurso en condiciones cultivadas. La FAO (2018), constantemente está mostrando su preocupación por la conservación de especies y variedades regionales de alto potencial agrícola que en algunos casos están siendo sub utilizadas y en otros, se ven desplazadas por otras de origen foráneo, que presentan generalmente una mayor demanda, capacidad productiva y rentabilidad económica. Hace énfasis en que el rico acervo genético de las variedades locales corre el riesgo de perderse y echar por tierra el esfuerzo ancestral de recolección, selección y desarrollo empírico.

El presente estudio trata de contribuir, aunque de manera limitada, evitando la pérdida de la biodiversidad de las legumbres locales que son parte de la soberanía alimentaria, que está en riesgo progresivo; por lo tanto, existe una profunda necesidad de revalorar y revalorizar el patrimonio genético de gran riqueza y los conocimientos ancestrales, a 
veces intangibles y subvalorados, como señala muy bien la FAO.

\section{CONCLUSIONES}

Las condiciones edafoclimáticas de la zona media del valle de Ica, distrito de Subtanjalla, en las que se desarrolló el cultivo de pallar, con los genotipos en estudio, permiten llegar a las siguientes conclusiones:

- Los doce genotipos de pallar evaluados mostraron buena adaptación a las condiciones de clima y suelo de la zona donde se llevó a cabo el estudio; sin embargo, las restricciones propias de la pandemia, ocasionaron importantes limitaciones en la etapa productiva.

- Se ha evaluado el comportamiento de doce genotipos de pallar de colores diversos, destacando los que tienen gran potencial productivo, al sobresalir en número de vainas por planta y número de granos por vaina.

- En el comparativo de rendimiento realizado, se ha determinado los genotipos más productivos, destacando los de color de grano variegado con guinda, granos de fondo gris, maculado con granate, y dos genotipos de color blanco y blanco cremoso.

- Se ha seleccionado interesantes segregantes a partir de los granos de colores que pasan a incrementar el germoplasma que se va recuperando.

- Se ha incrementado la cantidad de semilla y se encuentra disponible para la caracterización molecular y para compartir con el Banco de Germoplasma de INIA a fin de disminuir el riesgo de erosión genética del material ancestral.

\section{REFERENCIAS BIBLIOGRAFICAS}

Baudoin, J.-P, O. Rocha, J. Degreef, A. Maquet and L. Guarino. (2004). Ecogeography, Demography, Diversity and Conservation of Phaseolus lunatus L. in the Central Valley of Costa Rica. Systematic and Ecogeographic Studies on Crop Genepools 12. International Plant Genetic Resources Institute, Rome, Italy.

Ballesteros P. G., Torres G. A., Barrera M. Reincorporación del fríjol carauta (Phaseolus lunatus L.) a la agricultura tradicional en el resguardo indígena de San Andrés de Sotavento (Córdoba, Colombia). Disponible en: https://www.bioversityinternational.org/fileadmin/PGR/article-issue_123-art_4lang_es.html

Ccaccachahua, L. y Campos, C. (2007). Evaluación de caracteres morfo productivos de líneas avanzadas de pallar (Phaseolus lunatus L.) semi - precoz guiador en la 
zona media del valle de Ica. Tesis Ing Agrónomo U. N. "San Luis Gonzaga”. Ica. $66 \mathrm{p}$.

Debouck, D.G. (1999). Diversity in Phaseolus species in relation to the common bean.

In: "Common bean improvement in the twenty-first century", Singh, S.P. (ed.), Kluwer Academic Publ., Dordrecht, Holanda, pp. 25-52.

Espinoza, L. (2012). Asistencia técnica dirigida en Manejo y Sanidad en el cultivo de pallar. Guía Técnica. UNALM - AGROBANCO. 29 p.

Espinoza L. y Espino G. (2017). Caracterización morfológica y comparativo de rendimiento de 30 genotipos de pallar (Phaseolus lunatus L.) en la Zona Baja del Valle de Ica. Informe Final. Facultad de Agronomía. Universidad Nacional San Luis Gonzaga. Ica.

FAO. (2018). Nuestras legumbres: Pequeñas semillas, grandes soluciones. Ciudad de Panamá. 292 páginas. Licencia: CC BY-NC-SA 3.0 IGO.

FAO. (2018). Biodiversidad para una agricultura sostenible. El trabajo de la FAO sobre el uso de la biodiversidad en la alimentación y la agricultura. http://www.fao.org/3/CA2227ES/ca2227es.pdf

Fofana, E., DU Jardin, P. y J.P. Baudoin. (2001). Genetic diversity in the Lima bean (Phaseolus lunatus L.) as revealed by chloroplast DNA (cp DNA) variations. Genetic Resources and Crop Evolution. 48: 437-445.

Franco, T. L. e Hidalgo, R. (eds.). (2003). Análisis Estadístico de Datos de Caracterización Morfológica de Recursos Fitogenéticos. Boletín técnico no. 8, Instituto Internacional de Recursos Fitogenéticos (IPGRI), Cali, Colombia. 89 p.

Guimaraes, E; Ruane, J; Scherf, B; Sonnino, A; Dargie, J. (2007). Marker-assisted selection, current status and future perspectives in crops, livestock, forestry and fish. Food and Agricultural Organization of the United Nations, FAO. Roma, Italia. $471 \mathrm{p}$.

Hernandez-Villarreal, A. Caracterización morfológica de recursos fitogenéticos. Revista Bio Ciencias 2013; 2(3): 113-118.

IPGRI. (2001). Descritores para Phaseolus lunatus (Feijão-espadinho). International Plant Genetic Resources Institute, Rome. 51 p.

Jacinto-Hernández C., Garza-García R., Garza-García D. e I. Bernal-Lugo. Caracterización de germoplasma nativo de frijol con base en marcadores 
moleculares y atributos de calidad. Revista Mexicana de Ciencias Agrícolas Vol.5 Núm.2 15 de febrero - 31 de marzo, 2014 p. 253-264.

López-Alcocer, J., R. Lépiz-Ildefonso, D. González-Eguiarte, R. Rodríguez-Macías y E. López-Alcocer. Variabilidad morfológica de Phaseolus lunatus L. silvestre de la región occidente de México. Revista Fitotecnia Mexicana. Vol. 39 (1): 49 - 58, 2016.

Marrugo-Ligardo, Y.; Montero-Castillo, P.; Torregroza-Fuentes, E. y M. Duran-Lengua. (2012). Potencial nutricional de tres cultivares de frijol Zaragoza (Phaseolus lunatus L.) y estimación de su digestibilidad “in vitro”. Rev. Fac. Agron. (LUZ). 2012, 29: 314-326.

Maquet, A. (1995). Study on Genetic Diversity of the Legume Phaseolus lunatus Using Morphophysiological and Protein Markers. These de Doctorat. Gembloux, Faculté Universitaire des Sciences Agronomiques, Belgium, 188 p.

Milton J. S. (2007). Estadística para Biología y Ciencias de la Salud. McGraw-Hill Interamericana. 3ra. edición ampliada. 744 p.

Pesantes M., León E., De La Cruz, E. y J. Rodríguez. Variabilidad morfo-agronómica en poblaciones de pallar, Phaseolus lunatus, cultivado en condiciones de Costa de la Provincia de Trujillo (Perú). REBIOL 2015; 35(2): 29-38, Julio - Diciembre. 